\title{
Research of Information Interaction Simulation Method in Cooperative Vehicle Infrastructure System
}

\author{
Baigen Cai, Senior Member, IEEE, Congcong Wang, Wei ShangGuan, Member, IEEE \\ and Jian Wang, Member, IEEE
}

\begin{abstract}
The efficiency and performance of simulation method for information interaction process of Vehicular Ad-hoc Network (VANET) plays a crucial role in future Cooperative Vehicle Infrastructure System (CVIS). This paper puts forward a simulation method for information interaction process in CVIS based on the High Level Architecture (HLA) platform which consists of traffic simulator based on Paramics, network simulator based on OPNET and communication evaluation system in which evaluation indexes are integrated into the evaluation architecture through link-relative method. The simulation components act in close coordination with proposed time synchronization mechanism adaptively with feedback of their time differences. The test traffic scenario was built for the simulation method. The outstanding simulation results show that the time synchronization mechanism can shorten the time difference between Paramics and OPNET with proper step size, and the communication evaluation method we proposed in simulation platform can reflect the variation tendency of communication performance of VANET simulation system.
\end{abstract}

\section{INTRODUCTION}

In Cooperative Vehicle Infrastructure System (CVIS), the acquisition of vehicle information and roadside traffic information is realized through wireless communication and sensor technology, and it takes full advantage of the interaction between vehicles and roadsides to share information resources and realize intelligent transportation system. The main goals of CVIS are to achieve optimum utilizing traffic system resources, ensure passenger safety of vehicle driving and improve the traffic efficiency. Vehicular Ad-hoc Network (VANET) is the main information distribution way in information interaction process of CVIS with characteristics like multi-hop, dynamic topology structure, temporary and self-organization $[1,2]$. The widely applied and selectable communication modes in VANET include Wireless Local Area Networks (WLAN), $3^{\text {rd }}$ or $4^{\text {th }}$ Generation mobile communication, and Wireless Access in

This paper was supported by National Natural Science Foundation for Young Scholars of China (Grant No. 61104162), the State Key Program of National Natural Science Foundation of China (Grant No. U1334211), Fundamental Research Funds for the Central Universities of China (Grant No. 2013JBM007), the Scientific and Technology development program of China Railway Corporation (Grant No. 2013X009-D, 2013X003-B), Beijing Higher Education Young Elite Teacher Project (Grant No. YETP0538).

Baigen Cai, Congcong Wang, Wei ShangGuan and Jian Wang are with School of Electronic and Information Engineering and State Key Laboratory of Rail Traffic Control and Safety, Beijing Jiaotong University, Beijing, 100044, P. R. China. (e-mail: bgcai@bjtu.edu.cn; 12120280@bjtu.edu.cn; wshg@bjtu.edu.cn; wangj@bjtu.edu.cn)
Vehicular Environment (WAVE). Many kinds of transportation service have been applied by means of Vehicle-to-Vehicle and Vehicle-to-Infrastructure. Process of information interaction process in CVIS is shown in figure 1.

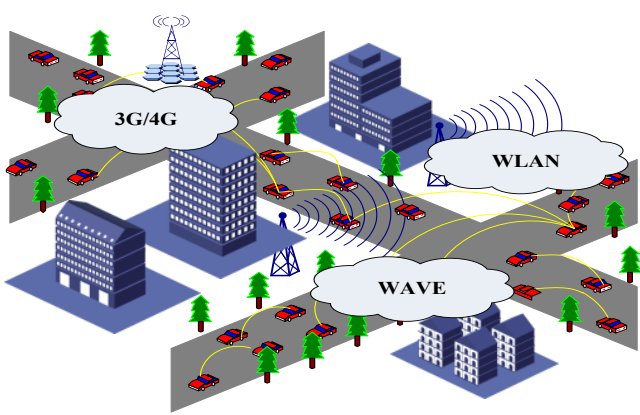

Figure.1. Schematic diagram of information interaction process in CVIS

The simulation model of information interaction in CVIS can be applied to test and verify a typical traffic scene like collision avoidance and provide a serviceable technical support and security driving recommendations for practical transportation system's technology research and development, especially in the area of automobile and traffic management. Information interaction process in CVIS shows supernormal complexity, so the conventional centralized simulation system is difficult to complete the traffic simulation and communication simulation simultaneously. In this paper, the collaborative simulation method based on High Level Architecture (HLA) is put forward. HLA platform coordinates the time sequence and data flow of traffic simulation and communication simulation synchronously through designed transmission interface and time synchronization mechanism.

The remainder of this paper is structured as follows. Section II gives a short survey on the current investigation of simulation method for VANET. Section III describes the proposed simulation method based on HLA in detail, especially the structure of simulation platform of information interaction, adaptive time synchronization mechanism and communication evaluation system. Implementation and verification for simulation method are presented in Section IV. Section V finally concludes this paper.

\section{RELATED WORK}

A key component for VANET simulations is a realistic vehicular mobility model that ensures conclusions drawn from 
simulation experiments will carry through to real deployments. In [3], author introduces a tool MOVE that allows users to rapidly generate realistic mobility models for simulations of VANET. Nowadays, many traffic simulators, such as Paramics, VISSIM, TraNS, VGSim, etc. provide vehicle mobility model and the ability to generate rode network [4]. In [5], author analyzes the category of vehicular mobility models, concludes the basic method to build vehicular mobility models, and introduces Suggested Upper Merged Ontology (SUMO) as a vehicular mobility model building software.

The simulation of communication is essence of VANET. The NCTUns network simulator/emulator is a powerful and valuable tool for network research and education. NCTUns supports two modes of vehicle mobility control, in addition, it also provide network simulator based on NS-2 which supports IEEE 802.11(p)/1609 protocol [6]. In [7], the paper proposes a comprehensive architecture for VANET simulation platform that focuses on producing reliable results. The architecture contains traffic and network simulators that communicate with each other in a dynamic and directional way. It adopts real-world data to model an activity-based traffic varying in time. The traffic generator aims at reproducing accurate vehicular traces for urban scenario. A higher level of realism can be obtained by modeling of human behavior with intelligent agents and by the implementation of related subsystems, like traffic management and control or weather factors. The distributed simulation traffic system can coordinate the function of every part flexibly to complete simulation of vehicle and communication as real as possible. In [8], authors favor the coupling of existing and well known simulators as we believe that the wireless communication characteristics influence the data transfer significantly and an oversimplified transmission model can lead to flawed results. In this paper authors describe the feedback loop between traffic and network simulators named traffic control interface and outline its versatility.

\section{SimUlation Method Of INFORMATION INTERACTION BASED ON HLA}

The simulation platform consists of three parts including traffic simulator based on Paramics, network simulator based on OPNET Modeler and communication evaluation system. In this simulation platform, traffic simulator sends vehicle and road network information to network simulator. At same time, communication evaluation system and traffic simulator focus on receiving communication results and the feedback of vehicle communication statuses from network simulator. Appropriate and adaptive time synchronization mechanism and communication evaluation method we proposed are applied in this simulation method.

\section{A. Structure of Simulation Platform Based on HLA}

HLA was issued by DMSO. The basic idea of HLA is to use object-oriented methods to design, develop, and realize the model object of the simulation system. In HLA, distributed member of simulation system with a particular simulation purpose is called "federal" and HLA architecture divides into parts which are Simulation Object Model (SOM), Federation Object Model (FOM) and Runtime Infrastructure (RTI). Federal members are the application of federal and they are the individual part of communication. Figure 2 illustrates the structure of simulation platform for information interaction process in CVIS based on HLA.

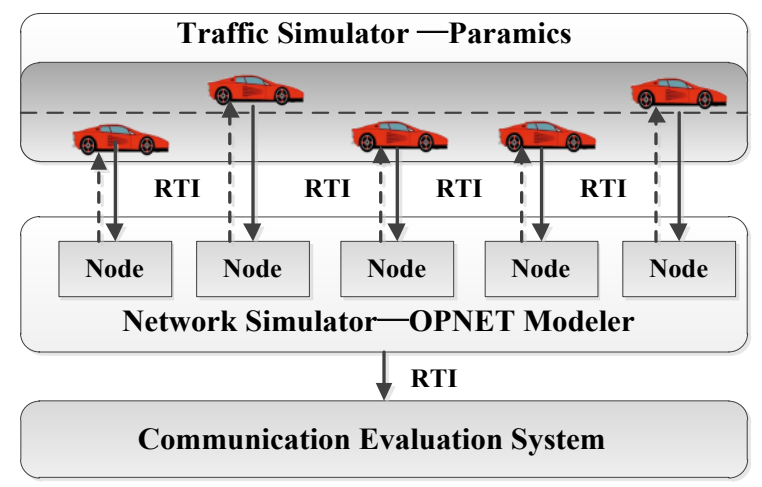

Figure.2. Structure of simulation platform for information Interaction process in CVIS based on HLA

HLA adopts "Publish/Subscribe" statement management mechanism to control the information interaction of federal members. Federal members can subscribe information data they are interested in. Data in the HLA exists in two forms as interaction class with timeliness and object class without timeliness. FOM defined the object class and interaction class which declare their properties and parameters of information. OPNET adopts interaction class to communicate with other federal members. In OPNET/HLA structure, the parameters of the interaction class of HLA corresponds to the properties of OPNET data packet, and the corresponding relationships of vehicle information in the Fed file and the Map file of OPNET are described in TABLE I in detail.

TABLE I

THE MAPPING OF INTERACTION ClASS AND DATA PACKET

\begin{tabular}{|l|l|}
\hline OPNET Packet & Paramics Vehicle Attitudes \\
\hline simulation_time & Parameter simulation_time \\
\hline vehicle_type & Parameter vehicle_type \\
\hline vehicle_id & Parameter vehicle_id \\
\hline latitude & Parameter latitude \\
\hline longitude & Parameter longitude \\
\hline velocity & Parameter velocity \\
\hline lane & Parameter lane \\
\hline roadside & Parameter roadside \\
\hline preceding_vehicle & Parameter preceding_vehicle \\
\hline following_vehicle & Parameter following_vehicle \\
\hline destination & Parameter destination \\
\hline communication & Parameter communication \\
\hline scene position & Parameter scene position \\
\hline
\end{tabular}

B. Traffic Simulation Based on Paramics

Besides having real dynamic 3D visualization for the user and the ability of micro-control for single vehicle, Paramics 
can also realize simulation of road system, real-time visualization simulation scenario, designing the interaction network, adaptive control of road signal and online or offline statistical analysis for simulation data. In simulation platform, Paramics regards as the traffic simulator, and its main function is generating road network and vehicle information and transferring them to network simulator with HLA interface. Simulation step size of Paramics ranges from $1 / 30 \mathrm{sec}$ to $1 / 2$ sec. The problem and challenge are that whether the simulation step size of traffic simulator is in step with the simulation time of the whole platform. The traffic simulation process based on Paramics is described in Figure 3.

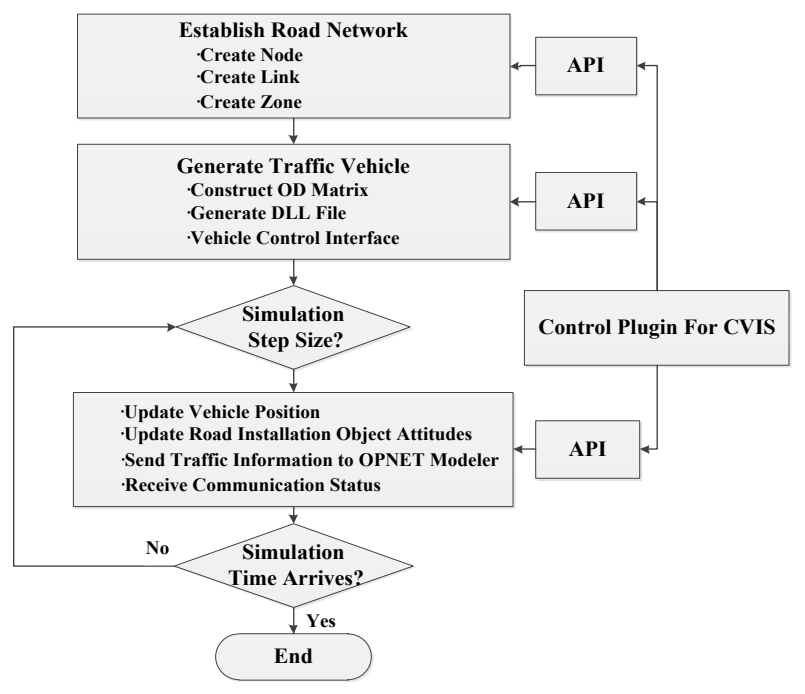

Figure.3. Traffic simulation process based on Paramics

\section{Network Simulation Based on OPNET}

OPNET Modeler adopts discrete event driven mechanism which is divided into four parts including the configuration of network topology, configuring business, collecting statistics, and running simulation. In consideration of the simulation technical limitations of OPNET Modeler and the simulation purpose which is verifying the feasibility of the information interaction simulation method, the following paper will emphasizes on simulation of WLAN as the communication mode of Vehicle Ad-hoc Network. The simulation study of multiple communication modes which exists the problem of mode switching is similar with signal mode. According to the particularity of information interaction process in CVIS, the MANET model of OPNET Modeler model library is selected as vehicle and roadside equipment simulation experiments. In addition, as the whole system is based on the platform of HLA, HLA interface node also should be joined and the node which control radio distance is also should be joined. A third-party application needs to assist the information transfer between HLA and OPNET Modeler.

MANET which is the main information carrier in VANET node plays a decisive role to the entire network environment simulation. The attitudes of MANET include Ad-hoc routing parameters and Wireless LAN parameters, etc. The network simulation process based on OPNET Modeler is described in the following steps.

Step 1: Receiving traffic data. The receiving traffic information includes the vehicle position information and vehicles which need making commutation with some other vehicles. MANET nodes modify their position attitudes and communicate with other nodes according the receiving HLA information through API provided by OPNET Modeler.

Step 2: Communication simulation. Vehicle node judges whether it requires communicating with other node according the "communication" attitude of packet in Table I. The "scene position" attitude of packet defines the position, in which area vehicle will make communicate and roadside equipment will broadcast traffic information. The range which center is "scene position" is one square kilometer. MANET adopts which kind of communication protocol according the proper modification of simulation attitudes. In the designed simulation platform, there is not a specific regulation, so it means communication between nodes happens randomly, and they don't have a given transfer destination.

Step 3: Sending simulation results. The sending HLA information packet contains the vehicle communication status and the communication performance results which includes transmission delay, packet loss rate and throughout. Vehicle communication status is received by traffic simulator Paramics and communication performance results are received by communication evaluation system.

\section{Communication Evaluation System}

Communication Evaluation System is used for stating and analyzing the wireless communication quality during the simulation process of information interaction in CVIS. The communication performance results including transmission delay, packet loss rate and throughout received from OPNET Modeler will be analyzed and evaluated through link-relative method. The essence of link-relative method is determining the weight of every indicator according their consequence and its process is specified as follow.

Step 1: Evaluation indexes are arranged in any order. In this platform, there are three communication evaluation indexes, which are $X_{1}, X_{2}, X_{3}$, representing transmission delay, packet loss rate and throughout respectively.

Step 2: Relative importance ratio $\lambda_{i}$ of adjacent indexes is calculated in turn. The calculation method of $\lambda_{i}$ in this paper is shown as following method.

if $X_{i}$ is more important (or less important) than $X_{i+1}$, then $\lambda_{i}=3($ or $1 / 3)$.

if $X_{i}$ is slightly important (or less important) than $X_{i+1}$, then $\lambda_{i}=2($ or $1 / 2)$.

if $X_{i}$ is equally important with $X_{i+1}$,

then $\lambda_{i}=1$. 
Step 3: Ensure reference value $\delta_{i}$, the reference value of the last evaluation index is set as 1 , then the reference value of other indexes can be benchmarked in order. The following formula shows the calculation process. $n$ is the mount of evaluation indexes, in other words, $n=3$.

$$
\delta_{i}=\lambda_{i} \delta_{i+1}, i=1,2,3, \cdots, n-1
$$

Step 4: $w_{i}$ is obtained by normalized processing of $\delta_{i}$ as formula (2), $n=3$.

$$
w_{i}=\frac{\delta_{i}}{\sum_{i=1}^{n} \delta_{i}}
$$

Through the link-relative method above, the weight of the communication evaluation indexes can be obtained reasonably and the TABLE II shows the calculation process and the value of $\lambda_{i}, \delta_{i}$ and $w_{i}$.

\section{TABLE II}

The Calculation Process Of The Value OF $\lambda_{i}, \delta_{i}$ and $w_{i}$

\begin{tabular}{|c|c|c|c|}
\hline $\begin{array}{c}\text { Evaluation } \\
\text { Index }\end{array}$ & $\lambda_{i}$ & $\delta_{i}$ & $w_{i}$ \\
\hline$X_{1}$ & 3 & 6.0 & 0.67 \\
\hline$X_{2}$ & 2 & 2.0 & 0.22 \\
\hline$X_{3}$ & - & 1.0 & 0.11 \\
\hline
\end{tabular}

In order to evaluate the communication performance comprehensively, $P_{e}$ integrates the transmission delay, packet loss rate and throughout with their weight as formula (3). The performance is better when the value of $P_{e}$ is higher. $t_{d}, p_{l}$ and $m_{t}$ represent transmission delay, packet loss rate and throughout and $t_{\max }, p_{\max }$ and $m_{\max }$ represent the maximum value of them.

$$
P_{e}=1-w_{1} \cdot \frac{t_{d}}{t_{\max }}-w_{2} \cdot \frac{p_{l}}{p_{\max }}+w_{3} \cdot \frac{m_{t}}{m_{\max }}
$$

\section{E. Adaptive Time Synchronization Mechanism}

The accuracy of time synchronization makes effects on the degree of simulation reality. The simulation time of traffic simulator and network simulator must have no difference. HLA architecture needs to maintain global correctness of time logic, therefore OPNET adopts the "Time Constrained" management strategy, namely the time advance of OPNET federal member is influenced by traffic simulator.

This paper proposed a time synchronization mechanism to coordinate simulation time between Paramics and OPNET Modeler. The simulation step size is related to simulation efficiency. The main essence of this method is adjusting the next step size of Paramics dynamically based on the feedback communication data from OPNET Modeler adaptively to ensure the run of simulation effectively. The steps of time synchronization mechanism are described as follow.
Step 1: Initialize simulation time. $S_{i}$ is the $i^{\text {th }}$ step size of Paramics and $T$ is simulation time of the platform at present.

$$
T=\sum_{i} S_{i} \text {, and } S_{1}=1 / 30
$$

Step 2: Calculate the $i+1^{\text {th }}$ step size. Traffic simulation suspended for $S_{i}$. Stat the timestamp $t_{i j}$ of communication status from OPNET Modeler, and $i$ is the times of time advance, $j$ is the ID of communication status. $m_{i}$ is the mount of communication statuses in the $i^{\text {th }}$ time advance. $\alpha$ is the proportional control factor for next step size as formula(5).

$$
\begin{gathered}
\alpha=\frac{T \cdot m_{i}}{\sum_{j} t_{i j}}, \alpha>1 \\
\text { if }\left(T-\frac{\sum_{j} t_{i j}}{m_{i}} \geq \frac{S_{i}}{2}\right) \\
\text { then } \quad S_{i+1}=\alpha \cdot S_{i} \\
\text { else } \quad S_{i+1}=\frac{1}{\alpha} S_{i} \\
\text { if } S_{i} \geq 0.5
\end{gathered}
$$$$
\text { then traffic simulation pauses. }
$$$$
\text { until } T-\frac{\sum_{j} t_{i j}}{m_{i}} \leq \frac{S_{i}}{2}
$$$$
\text { else go back to Step2. }
$$$$
\text { until simulation end. }
$$

The flow scheme of proposed time synchronization mechanism is shown in figure 4 .

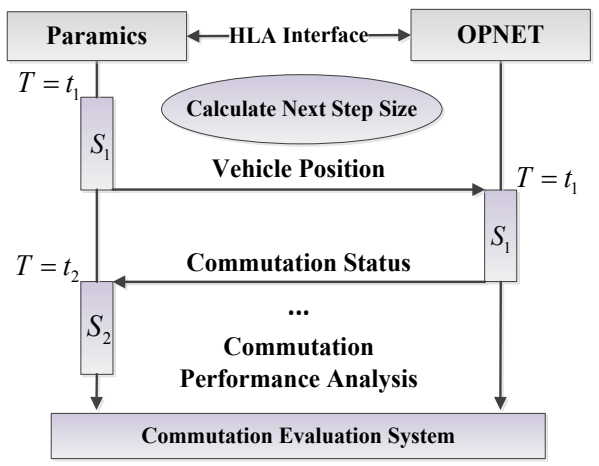

Figure.4. Process of time synchronization mechanism

\section{Test For Proposed Simulation Method}

The fundamental function of simulation method authors proposed is finishing simulation of information interaction process in CVIS precisely and timely through the simulation platform based on HLA. After implementation of simulation platform, test process goes by following aspects which including test for time synchronization mechanism and the communication evaluation system. 


\section{A. Implementation of Simulation Platform Based on HLA}

The federal members make communication with each other through RTI interface in HLA, so traffic simulator and network simulator must have a third part application to coordinate and control Paramics and OPNET Modeler respectively. The function of third part application for Paramics is controlling the generation of traffic information, step size of traffic simulation and the transportation of HLA information. At the same time, third part application for OPNET Modeler completes the transportation of traffic and communication information. The communication evaluation system is an application itself which receiving communication performance data from OPNET with HLA interface.

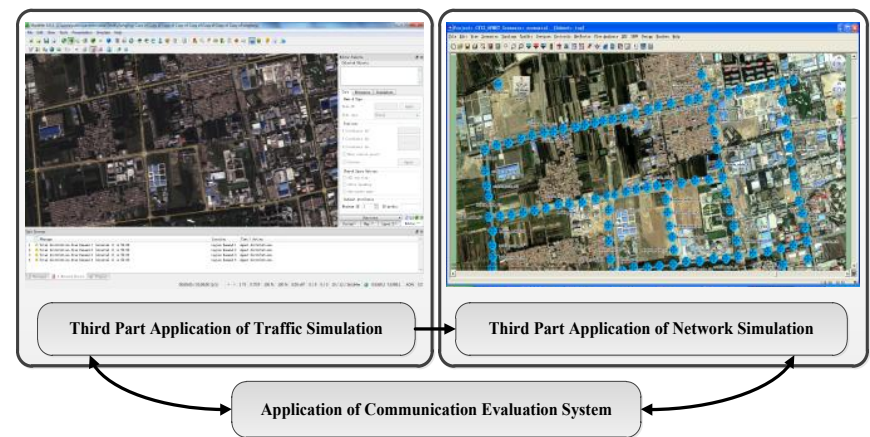

Figure.5. The components of information interaction simulation platform

The figure 5 shows the components of simulation platform. After defining the data interface of interaction class and object class and data transmission flow, the test traffic scenario is built in Paramics. As the test purpose is to verify the feasibility of the simulation method and stability of platform, there is not specific accident model in platform except the traffic flow information. The test scenario consists of 11 intersections in Lang fang of China and more than 500 vehicles which are distributed averagely in the road network. The velocity of vehicles is generated randomly in the normal range. Then the simulation can run normally as described above and the whole simulation lasts 30 minutes.

\section{B. Test for Time Synchronization Mechanism}

The method collects the timestamps of simulation components during simulation time. The time difference with several step size of traffic simulator is shown in figure 6 .

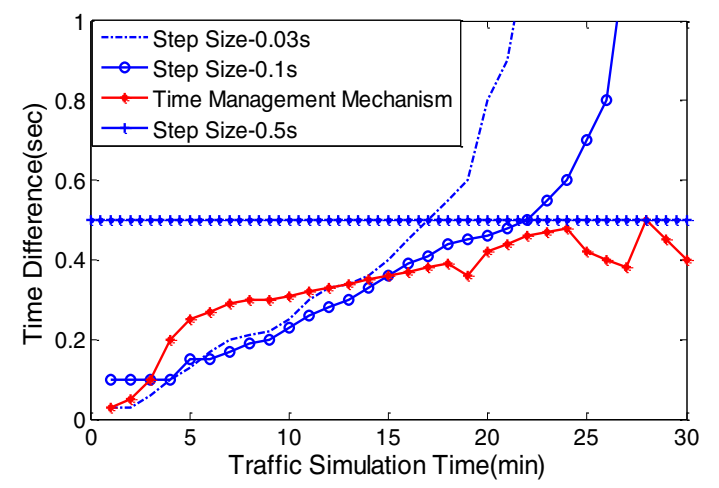

Figure.6. The time difference with different step sizes during simulation
The effective time synchronization mechanism means it can reduce the time difference among simulation federals. In this paper, the test indicator for time synchronization mechanism is the time difference between Paramics and OPNET Modeler. Figure 6 describes the variation tendency of time difference between traffic and network simulator, and the performance of time synchronization mechanism we proposed is optimal. If the step size is small relatively, the operational efficiency of network simulator can't reply the frequent communication response to traffic simulator, so it forms congestion as time goes on and the time difference becomes larger and larger. If the step size is large relatively, network simulator OPNET Modeler has enough time to make communication simulation, but instantaneity may be bad. The time synchronization mechanism can adjust the step size adaptively according to the current time difference, so its real-time performance is better than that with fixed step size.

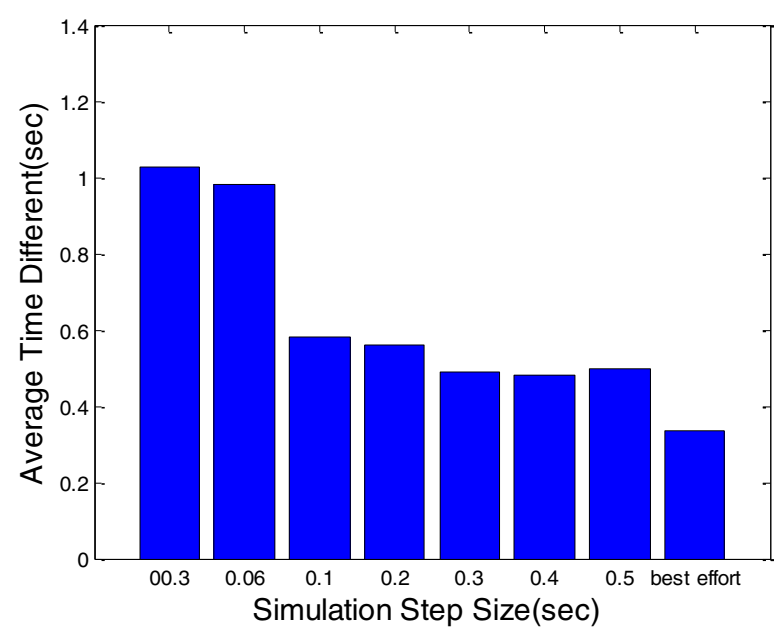

Figure.7. The average time difference with different step sizes

The average time difference in the whole simulation process can explain the overall effort of the adaptive time synchronization mechanism. Figure 7 show the average time difference with different step sizes. The average time difference of the proposed time synchronization mechanism with the best effort is $0.337 \mathrm{sec}$. The average time difference brings down along with the increase of step size basically.

\section{Communication Evaluation for Simulation Method}

As the paper described above, in the communication evaluation system, the weights of transmission delay, packet loss rate and throughout are $0.67,0.22$ and 0.11 respectively in communication evaluation system. The test purpose is to observe if the communication evaluation system is satisfied with change rule of the traffic flow and simulation strength. As the traffic flow is generated by random, the influence for the communication performance is low relatively. The congestion caused by small step size lead to the bad communication performance. The figure 8 and Tab III show the changing value and average value of communication performance factors with different step sizes. 

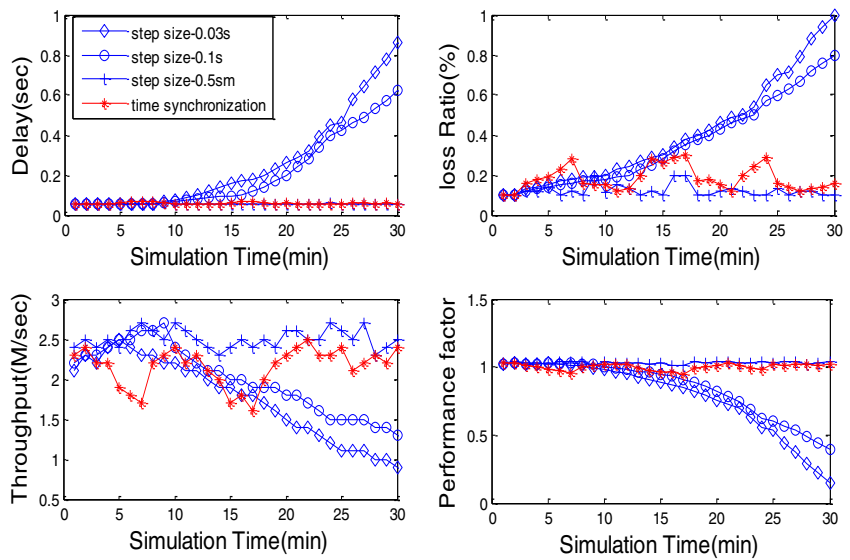

Figure.8. Communication performance indexes with different step sizes

TABLE III

The Average VAlue Of COMMunication PERFormance WITH DIFFERENT STEP SIZES

\begin{tabular}{|c|c|c|c|c|}
\hline $\begin{array}{c}\text { Step size } \\
(\mathbf{s e c})\end{array}$ & $\begin{array}{c}\text { Delay } \\
(\mathbf{s e c})\end{array}$ & $\begin{array}{c}\text { Loss rate } \\
(\mathbf{\%})\end{array}$ & $\begin{array}{c}\text { Throughput } \\
(\mathbf{M} / \mathbf{s e c})\end{array}$ & $\begin{array}{c}\text { Performance } \\
\text { factor }\end{array}$ \\
\hline 0.03 & 0.257 & 0.402 & 1.760 & 0.783 \\
\hline 0.06 & 0.237 & 0.373 & 1.982 & 0.802 \\
\hline 0.10 & 0.203 & 0.355 & 2.010 & 0.845 \\
\hline 0.20 & 0.176 & 0.312 & 2.292 & 0.860 \\
\hline 0.30 & 0.122 & 0.289 & 2.374 & 0.909 \\
\hline 0.40 & 0.672 & 0.243 & 2.421 & 0.986 \\
\hline 0.50 & 0.056 & 0.121 & 2.506 & 1.031 \\
\hline Best effort & 0.059 & 0.123 & 2.388 & 1.011 \\
\hline
\end{tabular}

The above results show that all the communication performance factors including transmission delay, packet loss rate and throughout deteriorate with the simulation step size's decrease progressively. The reason of that is OPNET Modeler can't finish communication simulation timely if step size is not appropriate, so more and more information accumulates, and then it leads to unsatisfied results. $P_{e}$ can embody the change rules and variation tendency of all the performance factors basically. The superiority of proposed time synchronization mechanism behaves obviously.

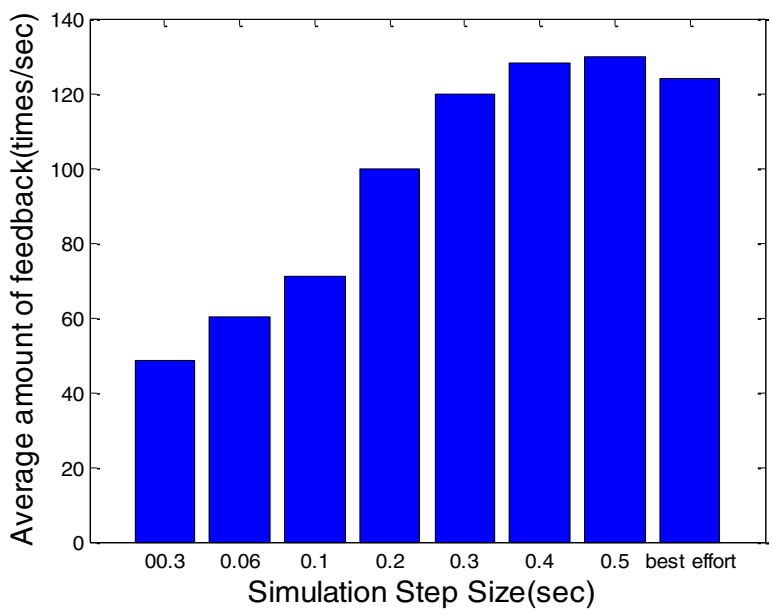

Figure.9. Amount of feedback communication data with different step sizes
The amount of feedback communication data from OPNET Modeler also is an indicator which can reflect the frequency of communication. When the message in platform transfers with congestion, the amount of feedback data will decrease rapidly. The figure 9 shows the different feedback amount of communication statuses with different step sizes. The change tendency of it is similar to $P_{e}$ and time difference. The communication performance described above states that time synchronization mechanism can coordinate simulation course steadily to get better communication performance and the communication evaluation system can analyze and assess the communication factors precisely.

\section{CONCLUSION AND FutURE WorK}

In this paper, according to analyze the characteristics of CVIS and its information interaction process, we proposed a simulation method for VANET based on HLA. The simulation method is embodied by the platform which consists of traffic simulator based on Paramics, network simulator based on OPNET Modeler and communication evaluation system in which several communication evaluation indexes, such as transmission delay, packet loss rate, throughput, are integrated into the evaluation architecture through link-relative method. Through dynamic accommodation of step size of traffic simulation, the proposed time synchronization mechanism can reduce the time difference between traffic simulator and network simulator effectively. The excellent test results from communication evaluation system also verify the feasibility of simulation method based on CVIS. In future works, more flexible VANET simulation methods with multiple communication modes based on different simulators and platforms will be investigated, and more accurate time synchronization mechanism and communication evaluation system will be focused.

\section{REFERENCES}

[1] A. Studer, F. Bai, B. Bellur, et al., "Flexible, extensible, and efficient VANET authentication," JCOMMUN NETW-S KOR, vol. 11, no. 6, pp. 574-588, Dec. 2009.

[2] H. Hartenstein, K. P. Laberteaux, "A tutorial survey on vehicular ad hoc networks," IEEE Commun. Mag, vol. 46, no. 6, pp. 164-171, Jun. 2008.

[3] F. K. Karnadi, Z. H. Mo, \& K. C. Lan, "Rapid generation of realistic mobility models for VANET," in Proc. IEEE WCNC, 2007, pp. 2506-2511.

[4] R. Stanica, E. Chaput, \& A. L. Beylot, "Simulation of vehicular ad-hoc networks: Challenges, review of tools and recommendations," Computer Networks, vol. 55, no.14, pp. 3179-3188, Jun. 2011.

[5] Z. Li, J. Hu, "Framework of Real VANET Simulation Research," in Proc.IEEE MINES, 2011, pp. 136-140.

[6] S. Y. Wang, C. C. Lin, "NCTUns 6.0: a simulator for advanced wireless vehicular network research," in Proc.IEEE VTC, 2010, pp. 1-2.

[7] A. Grzybek, M. Seredynski, G. Danoy, et al., "Aspects and trends in realistic VANET simulations," in Proc.IEEE WoWMoM, 2012, pp. $1-6$.

[8] A. Wegener, H. Hellbruck, C. Wewetzer, et al., "VANET simulation environment with feedback loop and its application to traffic light assistance," in Proc.IEEE GLOBECOM Workshops, 2008 , pp. 1-7. 